\title{
A classification of groups with a centralizer condition II: Corrigendum and addendum
}

\section{Zvi Arad and Marcel Herzog}

The aim of this note is to prove a theorem which extends the results of the authors' earlier paper, Bulz. Austral. Math. Soc. 16 (1977), 55-60. As one of the corollaries we prove Theorem 2 of that paper, the proof of which was incomplete.

We prove the following theorem.

THEOREM 1. Let $G$ be a finite group and let $M$ be a CC-subgroup of $G$. Denote the set of primes dividing $|M|$ by $\pi$. Then, either $O_{\pi}(G) \neq 1$ and

(1) $G$ is a Frobenius group with $M$ as the Frobenius kernel, or $O_{\pi}(G)=1$ and one of the following holds:

(2) $G$ is a Frobenius group with $M$ as a Frobenius complement;

(3) $G=o_{\pi^{\prime}}(G) N_{G}(M)$, a solvable group;

(4) there exists $H \triangleleft G$ satisfying

(a) $H \cap M \neq 1$, and

(b) $H / O_{\pi}(H)$ is simple.

Proof. If $G$ is simple, then clearly (4) holds. Thus assume that $N \neq 1$ is a minimal normal subgroup of $G$ and Theorem 1 is true for groups 
of order less than $|G|$.

Case 1. $N$ is a $\pi$-group. Then $N \subseteq M$ and consequently $V=\cap\left\{M^{a} \mid g \in G\right\} \supset 1$. If $V=M$ then $M \triangleleft G$ and (1) holds. So assume that $l \subset V \subset M$. As $V$ is a normal $C C$-subgroup of $M$ and of $G$, both $M$ and $G$ are Frobenius groups with the kernel $V$. Let $C$ be a complement of $V$ in $M$. Then $C$ is a $C C$-subgroup of $G$ and by [2, Lemma 1], $C V / V$ is a $C C$-subgroup of $G / V$, However, by [4, Theorem V, 8.18], $G / V$ has a nontrivial center, a cor.tradiction.

Thus we may assume that $o_{\pi}(G)=1$.

Case 2. $N$ is a $\pi^{\prime}$-group. In view of Lemma 1 in [2], $G / N$ sat.:sfiles the assumftions $6 . l^{\prime}$ Theorem 1 with respect to $M N / N$. By the inductive hypotehsis $G / N$ satisfies one of (1)-(4). Since $N$ is a nontrivial normal $\pi^{\prime}$-subgroup of $G, N_{G}(M)$ is solvable (see Theorem 2.3.h in [3]). Thus if $G / N$ satisfies (1), then by [2, Lemma 2] $G$ satisfies (3). If $G / N$ satisfies (2), then also $G$ satisfies (2) and if $G / N$ satisfies (3) then by [2, Lemma 2] so does $G$. Finally, if $G / N$ satisfies (4), then it is easy to see that so does $G$. Thus, in Case 2, the theorem holds.

Consequently we may assume that $O_{\pi}(G)=O_{\pi^{\prime}}(G)=1$. As $M$ is a Hall subgroup of $G$, it follows that $M \cap N \neq 1$ and since $M \cap N$ is a CC-subgroup of $N, N$ is simple. Thus (4) holds with $H=N$, and the proof of Theorem 1 is complete.

The theorem immediately yields

COROLLARY 1. If $G$ is solvable, then $G=o_{\pi},(G) N_{G}(M)$. We also have

COROLLARY 2. If $N_{G}(M)=M$, then $O_{\pi}(G)=1$ and either (2) holds or $O_{\pi},(G)=1$ and (4) holds with a simple $H$.

Proof. Suppose that (2) doesn't hold. It suffices to show that $L \equiv O_{\pi},(G)=1$. Otherwise, $M L$ is a Frobenius group with a complement $M$. Thus $Z(M) \neq 1 \quad[4$, Theorem $\mathrm{V}, 8.18]$ and $M$ is a TI-group [3, Theorem 2.1]. As $N_{G}(M)=M$, it follows that $G$ satisfies (2), a contradiction. 
As a final corollary we prove Theorem 2 of [2]:

THEOREM 2. If in Theorem 1, $N_{G}(M)=M$ and 3||$M \mid$, 'then either (2) holds or $G \cong \operatorname{PSL}(2, q)$ for some $q \geq 4$.

Proof. If 2||$M \mid$, then by [5], either (2) holds or $G \cong \operatorname{PSL}\left(2,2^{2 n}\right)$. Thus assume that 2||$M \mid$ and by Corollary 2 we may assume that ( 4 ) holds, with a simple $H$. In addition, we shall assume that Theorem 2 holds for groups of order less than $|G|$.

Case 1. $H \subset G$. If 3||$H \mid$, then $H \cong S z(q)$ by Thompson's classification of simple 3 '-groups, and if 3||$H \mid$, then $H \cong \operatorname{PSL}(2, q)$ for some $q$ or $H \cong \operatorname{PSL}(3,4)$ by [1, Theorem B]. In all cases $H$ has one class of involutions and all involutions of $M H$ belong to $H$. As $M$ is a CC-subgroup of $M H$ and 2$\}|M|$, counting of involutions forces $M \subset H$. By induction $H \cong \operatorname{PSL}(2, q)$ and as $N_{H}(M)=M, M$ is the normalizer of a Sylow group in $H$. Thus $G$-conjugates of $M$ are already conjugate in $H$ and counting conjugates of $M$ yields $H=G$, a contradiction.

Case 2. $H=G$. Thus $G$ is simple and $M$ satisfies 3||$M \mid$, $2\}|M|$, and $N_{G}(M)=M$. By [1, Theorem B], $G \cong \operatorname{PSL}(2, q), q$ odd. The proof of Theorem 2 is complete.

\section{References}

[1] Zvi Arad, "A classification of groups with a centralizer condition", Buzz. Austral. Math. Soc. 15 (1976), 81-85.

[2] Zvi Arad and Marcel Herzog, "A classification of groups with a centralizer condition II", BulZ. Austral. Math. Soc. 16 (1977), $55-60$.

[3] Marcel Herzog, "On finite groups which contain a Frobenius subgroup", J. Algebra 6 (1967), 192-221.

[4] B. Huppert, Endliche Gruppen I (Die Grundlehren der mathematischen Wissenschaften, 134. Springer-Verlag, Berlin, Heidelberg, New York, 1967). 
[5] Michio Suzuki, "Two characteristic properties of (ZT)-groups", Osaka Math. J. 15 (1963), 143-150.

Department of Mathematics,

Bar IIan University,

Ramat-Gan,

Israel;

Department of Mathematics, Institute of Advanced Studies, Australian National University, Canberra, ACT. 